\title{
Study on Evaluation of Coaches Training Efficiency Based on Competency Model and Super Efficiency DEA
}

\author{
Caiyan $\mathrm{Xu}$ \\ Jiangsu University \\ ntyzzj2004@163.com
}

\begin{abstract}
In the field of occupation sports, the coach is the compass. If there is no coaches for guidance, the athletes-training direction is not clear, the harder training, the more inefficient. If athlete lost the direction, quick action can be very dangerous. The important role of coaches is self-evident, but because of the special nature of the work of coaches, an athlete training success is difficult to accurately measure the efforts of athletes and coaches, which caused the coaches had mass phenomenon, while the occupation moral constraints coaches' behavior alone is insufficiency. Therefore, how to assess the quality and ability of coaches has become a problem in sports. Based on the study of the coaches competency model in the previous, and take the economics calculation method of the super efficiency DEA as data mining method, calculated by the computer, effectively reduce the complexity of the algorithm, directly linked the coach's competence and training outcomes and then calculate the training efficiency, to explore a method for considering the level of coaches.
\end{abstract}

Key words: Coach, Super efficiency DEA, Training efficiency, data mining

\section{Introduction}

As everyone knows, exercise training is a very complex system. In this system, the coach is the control body, and the athlete is the controlled object. They contact each other, and a mutual restriction, composing a training system. Zhu Shuchun thought: As the control body, coaches will play a controlling role in the whole process of training. They should make the goal of the training phase, select and apply the corresponding training methods, and objectively assess the athletes' status. As the object of athletes, they must be an energetic and creative individual, to realize coach`s each control process [1]. It is easy to see that, the coach is athlete's compass. For the athletes' growth influencing factors, besides the athlete's own talent, coach guidance is critical factor.

However, for the coacher's work is close interaction with athletes, it is easy to measure the growth of athlete's skill through a training performance, but the coaches' contribution is difficult to comment. Since modern times, the increasing level of competition sports and lacking of the coach work rules results a prominent contradiction. We can often see coach and athlete, accuse each other after a match, and the treatment is usually ambiguous. And in a 
defeat match, although the result is obvious, but the coach's responsibility and contribution is usually difficult to evaluate, resulting coaches work performance is difficult to accurately evaluate. Compared to athletes have clear reward and punishment measures, coaches` work only with occupation morals. In this context, with the development of the theory of competency of human resources, study on the coaches' competency becomes the research hotspot of sports academic.

Competency refers to potential, profound features of individual, which can distinguish a person, work in the outstanding performance or the mediocre. It is motivation, traits, self image, attitude, values, domain knowledge, cognitive and behavioral skills. It is an individual characteristic of anything that can be reliably measured or counted and can significantly distinguish excellent performance and general performance [2].

For the concept of competency, from the rise of competency characteristics in American since the twentieth century 70's, different scholars have different definitions of it. The earliest concept was proposed by Harvard University Professor McClelland, he thought, competency was knowledge, skill, ability, character or motivation that similar or related to the job, work performance or other important results in life [3].

Ron Zemke thought competency is difficult to define the term for the problem is from some differences in the basic procedures and the philosophy [4]. Chappell also thought, competency is a controversial concept, its meaning is determined by the people use the word. For the history of research on the competency is very short, so there is no authoritative definition of competency is recognized by the academy. With the competent research, there are 3 main schools, including cognitive competence school, behavioral competency school and generally qualified characteristics school [5-9].

Research on cognitive competence school mainly concentrated in the field of higher education. On the study of linguistics as the representative, study personnel related language competency. Behavioral competencies school mainly serving the training, instead of certification, its research results are widely used in North America. In twentieth Century 60's, North America launched teacher education movement based on occupational performance. In this movement, scholars identify the competency of outstanding teachers based on the analysis of teachers` task, and carry out occupational education, to improve the quality of teachers [10-13].

General competency characteristics school is concerned with what characteristics can make the occupation personnel to become a competent staff, focusing on the target (competency researches in China are also belonging to this school). The late 80's to 90's of twentieth Century, many countries established national occupation certification system on the basis of the theory of competency, such as the British national occupational qualification system. New Zealand and USA launched the national skill standards. Research and application of competency are just started in our country. In 1990, 3 private enterprises in Hong Kong took the lead in the use of competency method to increase their management performance [14]. 
Shi Kan and Wang Jicheng from Chinese Academy of Sciences were modeled and evaluated competency of Chinese communication industry executives through behavioral event interview [15].

Professors Song Chanrong and Ling Wenquan were investigated middle managers of various industries and enterprises in 12 cities with the method of questionnaire investigation, through the data to establish the competency model of middle-level managers in China [16].

Competency research in the sports organizations started relatively late. In 2007, Liu Liu studied competency and competency model of professional sports coaches in China [17, 18]. In 2010, Zhang Yunliang studied performance appraisal based on competency of coaches [19]. In $2011 \mathrm{Li}$ Lin proposed a two order five factor model of job performance structure of professional sports coaches in China, for the order factor is job performance, other four factors are task performance, contextual performance, development performance and counter-productive performance. Their studies also found, the relationship performance on job performance contribution rate was greater than the other 3 factor contribution rate [20].

In 2011Tang Yongqiang studied the evaluation system of Guangdong youth soccer coaches, according to the "competency - duty performance" structure to build a competency model. It contains 9 two level indexes and 43 three level indexes. [21]

From the above study at home and abroad is not difficult to see, although the competency of sports coaches has become a hot research of sports, but for it has just started, the proposed competency model is different, and for the competency characteristics, these models are difficult to use in practical work.

In this context, based on the previous research of competency, this paper introduced super efficiency DEA algorithm, which often applied in economics, as data mining method to find the coaches training efficiency, and to advance research on the competency of coaches.

This paper is structured as follows:

The first section, introduction to the domestic and foreign research on the competency and necessity of the research issues;

The second section, the relevant theory and application of DEA;

The third section, the theory and application of super efficiency DEA;

The fourth section uses the super efficiency DEA algorithm to construct the coaches training efficiency model.

\section{DEA}

The productive activities and social activities people often encounter this problem: after a period of time, need to evaluate the departments. The evaluation is built on the "input" and "output" data of the decision making unit. The input data is some consumption that decision-making unit needs in some activities. For example, the total funds invested, total labor input number, covers an area and so on; the output data is the amount of information that decision-making unit make after some effective activities 
with certain input. For example, the number of different types of products, the quality of the products, the economic benefit and so on.

Data envelopment analysis is another research field of operations research. The first successful application of DEA is Charles and Cooper's case, the evaluation of public schools for mentally retarded children. In the evaluation, the output includes "index self-esteem" and other intangible; the input consists of parental care and education level of parents, it is easy to set the appropriate weights, which is also one of the advantages of DEA.

In 1978, the famous strategist A. Charms, W. W. Cooper and E. Rhodes proposed a method called data envelopment analysis, to evaluate the relative effectiveness between departments. Their model was named CCR model. This model is a very fruitful method to study with multiple inputs, especially with multiple outputs problem.

In 1984 R. D. Banker, A. Charles and W. W. Cooper proposed BCC model. In 1985 Charles, Cooper and B. Golany, L. Seiford, J. Stutz gives another model (called CCGSS model), the two models are used to study the production sector. In 1986 Charles, Cooper and Wei Quanling estimated the CCW model. In 1987, Charles, Cooper, Wei Quanling and Huang Zhimin proposed the model which has been called data envelopment model -- CCWH model. This model can be utilized to deal with the input and output of the situation, and the cone selection can reflect the decision maker's "preference". These models and the new model are being constantly improved and further developed.

Some of the models mentioned above can be regarded as a method for processing the multi objective decision making problem with multiple inputs and multiple outputs. Data envelopment analysis can be regarded as a new method for analyzing statistics. It is a method to observe applicable production frontier based on a set of input-output. In economics and econometrics, estimation of operative production frontier, commonly used statistical regression and other statistical methods. These estimates the production function established by these methods are non effective. For these confused estimation with the effective decision making units and non effective decision making units. In the evaluation of the effectiveness, in addition to the DEA method, there are some additional methods, but those methods are almost limited to the single output. In contrast, the DEA method dealing with the multi input, especially the multi output problem, with absolute advantage. Moreover, the DEA method can be utilized not only in linear programming but also can obtain much useful management information. Therefore, it is utilized very widely.

The benefits of DEA attracted numerous applications. Application scope has been expanded to flight, repair and maintenance of American military aircraft, and the army conscription, city, bank etc... The application field of this method is continuously expanding. It can also be used to study the relative efficiency between different schemes. DEA model can even be used to assess policy.

The most attractive study is to compare the DEA with other evaluation methods, for example, the application of DEA in the evaluation of the effectiveness of the hospital in North 
Carolina. The production function was given by the econometric regression method had been considered the returns to scale did not exist in this case. The DEA study found that, despite the use of the same data, production return function cannot be determination of returns to scale as well as the DEA.

The DEA method and the model, and the DEA approach to understanding and application are developing continuously and deeply. In addition to BCC, CCGSS, CCW model and CCWH model mentioned above, when the DEA method is used in the concrete, for example "window analysis" method, the application scope of the DEA is extended to the dynamic case.

Assume a set of the inspected unit number is $n$, each inspected unit with $S$ output and $M$ input variables. $Y_{j k}$ is the first k was inspected unit the $\mathrm{J}$ output variaples, $X_{i k}$ is the first k unit of the I input variables. Calculation of the $K$ decision units' total efficiency can be transformed into the linear programming problem:

$$
\begin{aligned}
& \min \theta \\
& \text { s.t. } \sum_{j=1}^{n} X_{j} \lambda_{j} \leq \theta X_{h} \\
& \sum_{j=1}^{n} Y_{j} \lambda_{j} \geq Y_{k} \\
& \lambda_{j} \geq 0, j=1,2, \cdots, n
\end{aligned}
$$

$X_{k}=\left(x_{1 k}, x_{2 k}, \cdots x_{m k}\right), Y_{k}=\left(y_{1 k}, y_{2 k}, \cdots y_{m k}\right)$ In CCR model, this model is obtained under the assumption of constant returns to scale. The efficiency of $k$ observed values of decision-making units, meets $0 \leq \theta \leq 1$. Its economic meaning is: all $k$ decision-making unit output of a linear combination can be replaced by decision making unit output $Y$ case, it can compression for the degree of $X$, The compression ratio of the size of $\theta$, the $\theta$ is also known as the efficient measure. When $\theta=1$, the inspected unit is the efficiency frontier points, in the state of efficiency. When the $\theta<1$ indicates that no efficiency, $1-\theta$ were the ratio of investment of the $k$ inspected unit to reach the efficiency frontier, which is the biggest proportion of investment can be reduced.

However, from the basic DEA model can be found, the all DMU result will be simply divided into two groups, a set of efficient DMU and the formation of Pareto boundary. The other group belongs to the invalid rate DMU. For these effective unit, if continue to evaluate, the model is incapable of action. In view of this situation, in order to distinguish the efficiency of decision making units, Andersen and PE .Tersen, put forward the construction of super efficiency DEA model to measure the so-called super efficiency, the efficiency of decision making units to sort on the frontier, and TONE thought it that can be removed with the efficiency of decision making units, to determine the difference between super efficiency. [21-24]

\section{Super Efficiency DEA}


A weakness of the traditional DEA model is the decision-making unit can only divide into effective and non effective two classes. It is incapable for these effective units continue to evaluate. A super efficient evaluation model was proposed by ANDERSON et al in 1993. It can sort the DEA efficient of decision making units. The super efficiency DEA model's (denoted as SE-DEA) different from the general DEA model is that it does not contain the evaluated reference concentration unit itself, this model is briefly introduced following.

Super-efficient evaluation mathematics model is similarity with the DEA model. Its form is as follows:

$$
\begin{aligned}
& \min \theta \\
& \text { s.t. } \sum_{j=1}^{n} X_{j} \lambda_{j} \leq \theta X_{h} \\
& \sum_{j=1}^{n} Y_{j} \lambda_{j} \geq Y_{k} \\
& \lambda_{j} \geq 0, j=1,2, \cdots, n
\end{aligned}
$$

Here the symbolic is significant as before. The difference with the front is, the basic idea is to evaluate $k$ efficiency of decision making units, the $k$ decision-making unit of input and output is a linear combination of all the other decision making unit of input and output substitution, and the $k$ decision-making unit excluded, while in front of the model that the decision making unit is included. Since evaluated unit efficiency value is decided by the effective unit (determined by the decision unit of the hyper plane.), so the no effect decision units in CCR model and in the super efficient model are same.

Theorem 1 decision unit $D M U_{o}$ CCR relative efficiency value is less than the sufficient and necessary condition of 1 is the super efficiency value is less than 1

Theorem 2 decision unit $D M U_{o}$ is weak CCR efficient (inactive) necessary and sufficient conditions of the super efficient model is weakly efficient (inactive).

Proofs see Ref. The model is the super efficiency DEA model with constant returns to scale; we also can get the super efficiency DEA model with variable returns to scale, as shown below:

$$
\begin{aligned}
& \min \theta \\
& \text { s.t. } \sum_{j=1}^{n} X_{j} \lambda_{j} \leq \theta X_{0} \\
& \sum_{j=1}^{n} Y_{j} \lambda_{j} \geq Y_{0} \\
& \sum_{j=1}^{n} \lambda_{j}=1 \\
& \lambda_{j} \geq 0, j=1,2, \cdots, n \neq 0
\end{aligned}
$$


Theorem 3 the relative efficiency of decision making units $D M U_{o}$ CCGSS less than 1is necessary and sufficient conditions for the super efficiency CCGSS relative efficiency is less than 1

Theorem 4 decision making unit $D M U_{o}$ is weak CCGSS efficient (inactive) if and only if the super efficiency CCGSS weakly efficient (inactive).

Proof sees Ref... Since Andersen proposed super efficiency DEA model in 1993, countless scholars have conducted in-depth research on relevant theory. Below we mainly introduced the relationship between the super efficiency DEA model and the returns to scale.

According to the meaning of returns to scale, it not only depends on the production function, but is also dependent on the production possibility set. So, in general, different production possibility set, the decision making unit returns to scale the situation is very different. So, the DEA model determines the returns to expand.

BANKER, THRALL has been put forward by the following theorem judging unit is in increasing returns to scale and decreasing state.

Theorem 5 decision unit $D M U_{o}$ in increasing returns to scale, when and only when the super efficiency DEA (CCR) all the optimal solution of the model to meet the $\sum_{j=1}^{n} \lambda_{j}^{*} \prec 1$ decision making unit $D M U_{o}$ in decreasing return to scale, when and only when the super efficiency DEA (CCR) all the optimal solution of the model satisfies $\sum_{j=1}^{n} \lambda_{j}^{*} \succ 1$.

Theorem 6 if the decision unit $D M U_{o}$ is decreasing returns to scale, the super efficiency DEA to output oriented (BCC) model is feasible, and the $\sigma^{*} \prec 1, \sigma^{*} \quad$ is the super efficiency DEA to output oriented (BCC) model of the optimal solution.

Proof: SE-BCC model with output oriented

$$
\begin{aligned}
& \sigma^{*}=\max \sigma \\
& \text { s.t. } \sum_{j=1}^{n} X_{j} \lambda_{j} \leq \theta X_{0} \\
& \sum_{j=1}^{n} Y_{j} \lambda_{j} \geq Y_{0} \\
& \sum_{j=1}^{n} \lambda_{j}=1 \\
& \sigma, \lambda_{j} \geq 0, j=1,2, \cdots, n \neq 0
\end{aligned}
$$

$\theta=\frac{1}{\sigma}$, the model is transformed into: 


$$
\begin{aligned}
& \min \theta \\
& \text { s.t. } \sum_{j=1}^{n} X_{j} \lambda_{j}^{*} \leq \theta X_{0} \\
& \sum_{j=1}^{n} Y_{j} \lambda_{j}^{*} \geq Y_{0} \\
& \sum_{j=1}^{n} \lambda_{j}^{*}=\theta=\frac{1}{\sigma} \\
& \sigma, \lambda_{j} \geq 0, j=1,2, \cdots, n \neq 0
\end{aligned}
$$

Here $\lambda_{j}^{*}=\theta \lambda_{j}$,

Since $D M U_{o}$ is decreasing returns to scale, by theorem 4, meet the condition of the optimal solution of all $\sum_{j=1}^{n} \lambda_{j}^{*} \succ 1$ is a feasible solution of the SE-CCR model.

$$
\begin{aligned}
& \min \rho \\
& \text { s.t. } \sum_{j=1}^{n} X_{j} \lambda_{j}^{*} \leq \rho X_{0} \\
& \sum_{j=1}^{n} Y_{j} \lambda_{j}^{*} \geq Y_{0} \\
& \rho, \lambda_{j} \geq 0, j=1,2, \cdots, n \neq 0
\end{aligned}
$$

Make $\sum_{j=1}^{n} \lambda_{j}^{*}=\theta$. Obviously, the $\theta>\rho$ is a feasible solution. That is to say $\lambda_{j}^{*}(j \neq 0)$ and $\theta$ is the model feasible solution.

Theorem 7 if the feasible solutions of SE-BCC model with output oriented exist, the evaluation decision unit $D M U_{o}$ is a constant returns to scale or the returns to scale (also known as non decreasing returns to scale).

Theorem 8 the extreme efficient $D M U_{o}=\left(x_{0}, y_{0}\right)$, if the production guide the returns to scale of non super efficiency DEA model to decline a solution, if and only if the decision unit $x_{0}, \delta y_{0}($ any $0 \prec \delta \leq 1)$ under BCC model is effective.

From the above analysis, under variable returns to scale condition, the super efficiency DEA model may not have feasible solution. Therefore cannot fully sort the effective unit.

With $\mathrm{N} D M U_{o}$, input $M$, output elements as $S, \quad X_{j}=\left(x_{1 j}, x_{2 j}, \cdots, x_{m j}\right)^{T}$ as input data set, $Y_{j}=\left(y_{1 j}, y_{2 j}, \cdots, y_{s j}\right)^{T}$ for the output data set, $j=1,2, \cdots, n$. When evaluating the $j_{0}$ decision making unit, VRS super-efficient model based on input as: 


$$
\begin{aligned}
& \min \theta \\
& \text { s.t. } \sum_{j=1}^{n} X_{j} \lambda_{j} \leq \theta X_{0} \\
& \sum_{j=1}^{n} Y_{j} \lambda_{j} \geq Y_{0} \\
& \sum_{j=1}^{n} \lambda_{j}=1 \\
& \theta, \lambda_{j} \geq 0, j=1,2, \cdots, n \neq 0
\end{aligned}
$$

If the evaluation $D M U_{o}$ has an output value is greater than the other decision making units, there is $y_{k 0} \succ y_{k j}(1 \leq k \leq s, j=1,2, \cdots, n, j \neq 0)$, the model has no feasible solution. Because the datacentralized decision making unit always exists, so the model can't sufficiently to rank effective $D M U$. In order to overcome the model non feasible solutions, we put the constraint conditions $\sum_{j=1}^{n} \lambda_{j}=1$ to $\sum_{j=1}^{n} \lambda_{j}=d \geq 1$, and then get the improved model:

$$
\begin{aligned}
& \min (\theta+M d) \\
& \text { s.t. } \sum_{j=1}^{n} X_{j} \lambda_{j} \leq \theta X_{0} \\
& \sum_{j=1}^{n} Y_{j} \lambda_{j} \geq Y_{0} \\
& \sum_{j=1}^{n} \lambda_{j}=d \geq 1 \\
& \theta, \lambda_{j} \geq 0, j=1,2, \cdots, n \neq 0
\end{aligned}
$$

Here $M$ is a large enough constant, can take $M=10^{6}$.

Due to the $M$, which forces the optimal values of the model $d^{*}$ to satisfy the constraints of minimum D. Obviously, there is always the optimal solution of the model.

Do the following transformation of the model: $\lambda_{j}=\frac{\lambda_{j}}{d^{*}}, y_{0}=\frac{y_{0}}{d^{*}}$, we have:

$$
\begin{aligned}
& \min \frac{\theta}{d^{*}} \\
& \text { s.t. } \sum_{j=1}^{n} x_{j} \lambda_{j} \leq \frac{\theta}{d^{*}} x_{0} \\
& \sum_{j=1}^{n} y_{j} \lambda_{j} \geq \frac{y_{0}}{d^{*}}=y_{0} \\
& \sum_{j=1}^{n} \lambda_{j}=1 \\
& \theta, \lambda_{j} \geq 0, j=1,2, \cdots, n \neq 0
\end{aligned}
$$

This model is a efficiency evaluation model of virtue $\operatorname{DMU}_{0}\left(x_{0}, y_{0}\right)$. 
Through the above analysis, this model has a feasible solution, and the optimal values are the efficiency of virtue $D M U_{0}\left(x_{0}, y_{0}\right)$ that is super efficiency is higher than the $D M U_{0}$ in output.

Through the above analysis, we can get:

Definition 1 the improvement based on the variable returns to scale for the super efficiency model is the evaluation of $D M U$, super efficiency value is $\left(\frac{\theta^{*}}{d^{*}}+d^{*}\right)$ (all $d^{*}, \theta^{*}$ are the optimal solution of models.

From the above definition we can see, for the effective decision making units optimal solutions of the model is $\min d=d^{*}=1$, that is to say, the model virtual $D M U$. Super efficiency definition for each effective $D M U$ efficiency values are enhanced by a unit. We give $D M U$ theorems of returns to scale

Theorem 9 to any optimal value $\mu_{0}^{*}$ :

(1) $D M U$ for the increasing returns to scale, when and only when the $\mu_{0}^{*} \succ 0$

(2) $D M U$ for the constant returns to scale, when and only when the $\mu_{0}^{*}=0$

(3) $D M U$ for diminishing returns to scale, when and only when the $\mu_{0}^{*} \prec 1$

For the super efficient model based on output of variable returns to scale, there is no feasible solution is due to the evaluated unit $D M U$ may have small input unit. Therefore, outputs of the VRS super efficiency model is given based on

$$
\begin{aligned}
& \max (\rho+\varepsilon d) \\
& \text { s.t. } \sum_{j=1}^{n} x_{j} \lambda_{j} \leq x_{0} \\
& \sum_{j=1}^{n} y_{j} \lambda_{j} \geq \rho y_{0} \\
& \sum_{j=1}^{n} \lambda_{j}=d \leq 1 \\
& \rho, \lambda_{j} \geq 0, j=1,2, \cdots, n \neq 0
\end{aligned}
$$

Here $\varepsilon>0$ is a small constant, can take $\varepsilon=10^{-6}$. In the model, because of very small and constant existence of $\varepsilon>0$, forcing the $d$ value is the maximum value to satisfy the constraints. This leads to a virtue $D M U$.

Definition 2 in the improved super efficiency model based on output with variable scale, Evaluation the efficiency value of $D M U$ to $\left(\frac{d^{*}}{\rho^{*}}+\frac{1}{d^{*}}\right) \quad\left(\right.$ all $d^{*}, \rho^{*}$ are optimal solution of the model). [25-29] 


\section{Study on Evaluation of Coaches Training Efficiency based on Competency Model and Super Efficiency DEA}

\subsection{Select Input Parameters}

The super-efficient DEA model is a kind of economic model. Its principle is classify the variable as input and output index, then construct the model to calculate the system efficiency. Although this study is about the training efficiency of coaches, but also can take coach competency as input indicators, the athletes' level as the output index, to study training efficiency based on coaches competency.

As mentioned above, the input indexes for the super efficiency DEA model are the coaches' competency, which proposed by Chengdu Sport University professor Liu Liu:

The acquisition of knowledge $C_{1}$, Team consciousness $C_{2}$, Believe team members and promote teamwork $C_{3}$, Authority $C_{4}$, Self-confidence $C_{5}$, Attention to detail $C_{6}$, and Care $C_{7}$.

The indicators described in Ref [17], in this paper, in order to quantify the competency for numerical super efficiency DEA input, selected 10 expert score of 20 professional coaches competency, average the scoring results obtained as Table 1:

Table 1. Scoring Results

\begin{tabular}{|c|c|c|c|c|c|c|}
\hline$C_{1}$ & $C_{2}$ & $C_{3}$ & $C_{4}$ & $C_{5}$ & $C_{6}$ & $C_{7}$ \\
\hline 69 & 72 & 79 & 85 & 90 & 96 & 87 \\
\hline 66 & 86 & 66 & 84 & 52 & 97 & 84 \\
\hline 86 & 89 & 85 & 78 & 75 & 96 & 88 \\
\hline 64 & 76 & 76 & 79 & 42 & 82 & 56 \\
\hline 73 & 84 & 72 & 79 & 76 & 83 & 88 \\
\hline 84 & 87 & 83 & 86 & 67 & 98 & 76 \\
\hline 83 & 73 & 64 & 83 & 54 & 96 & 89 \\
\hline 68 & 75 & 71 & 77 & 71 & 74 & 73 \\
\hline 89 & 71 & 68 & 75 & 57 & 94 & 82 \\
\hline 87 & 89 & 83 & 84 & 97 & 40 \\
\hline
\end{tabular}




\begin{tabular}{|l|l|l|l|l|l|l|}
\hline 69 & 82 & 96 & 84 & 74 & 96 & 71 \\
\hline 72 & 81 & 72 & 84 & 89 & 96 & 82 \\
\hline 87 & 84 & 77 & 72 & 79 & 77 & 37 \\
\hline 84 & 60 & 78 & 40 & 58 & 71 & 38 \\
\hline 85 & 65 & 82 & 83 & 63 & 97 & 74 \\
\hline 76 & 75 & 86 & 84 & 78 & 96 & 60 \\
\hline 68 & 90 & 90 & 45 & 83 & 88 & 67 \\
\hline 80 & 64 & 63 & 77 & 63 & 94 & 63 \\
\hline 81 & 82 & 87 & 74 & 74 & 40 & 91 \\
\hline 66 & 84 & 76 & 84 & 92 & 57 & 76 \\
\hline
\end{tabular}

\subsection{Select Output Indexes}

In order to objectively consider the coaches training efficiency, this paper selected 10 coaches` training records as the object of study, consider the experts and the existing research results, take some indexes of the training athletes at the training efficiency output index,

These indicators include: athletes number $P_{1}$, national event score (per person: gold 5 points, silver 4 points, Bronze 3 points, final 2 points) $P_{2}$, provincial tournament score ( per person: gold4 points, silver 3 points, Bronze 2 points, final 1 points) $P_{3}$, international event score (per person : gold 20 points, silver 16 points, bronze 10 points, final 4 points) $P_{4}$. The output index data are as Table 2:

Table 2. Output Index Data

\begin{tabular}{|c|c|c|c|}
\hline$P_{1}$ & $P_{2}$ & $P_{3}$ & $P_{4}$ \\
\hline 21 & 73 & 120 & 8 \\
\hline 30 & 100 & 110 & 20 \\
\hline 47 & 90 & 86 & 10 \\
\hline 20 & 77 & 70 & 16 \\
\hline 19 & 85 & 73 & 8 \\
\hline
\end{tabular}




\begin{tabular}{|c|c|c|c|}
\hline 23 & 88 & 48 & 8 \\
\hline 17 & 74 & 65 & 16 \\
\hline 16 & 76 & 72 & 8 \\
\hline 19 & 72 & 68 & 8 \\
\hline 13 & 88 & 90 & 8 \\
\hline 22 & 83 & 97 & 8 \\
\hline 11 & 82 & 70 & 20 \\
\hline 10 & 130 & 210 & 36 \\
\hline 24 & 61 & 78 & 8 \\
\hline 18 & 66 & 72 & 0 \\
\hline 18 & 76 & 87 & 26 \\
\hline 25 & 110 & 160 & 0 \\
\hline 30 & 65 & 64 & 8 \\
\hline 10 & 83 & 88 & 8 \\
\hline 20 & 85 & 77 & \\
\hline
\end{tabular}

\subsection{Coaches Training Efficiency Calculation}

According the use of SE-DEA to calculate the coaches' efficiency value, as showed in Table 3:

\section{Table 3. The Coaches' Efficiency Value}

\begin{tabular}{|c|c|c|c|}
\hline coach & Efficiency value & coach & Efficiency value \\
\hline coach 1 & 0.8929 & coach 11 & 0.6891 \\
\hline coach 2 & 1.9525 & coach 12 & 0.6712 \\
\hline coach 3 & 0.8714 & coach 13 & 2.9802 \\
\hline coach 4 & 0.8072 & coach 14 & 0.6891 \\
\hline coach 5 & 0.7494 & coach 15 & 0.7065 \\
\hline coach 6 & 0.6712 & coach 16 & 0.8001 \\
\hline coach 7 & 0.7596 & coach 17 & 1.9321 \\
\hline coach 8 & 0.7562 & coach 18 & 0.6432 \\
\hline coach 9 & 0.7648 & coach 19 & 0.5312 \\
\hline coach 10 & 0.7782 & coach 20 & 0.7596 \\
\hline
\end{tabular}

\section{Conclusions}

In this paper, the coaches' competency as the starting point, use economic modeling methods: super efficiency DEA method to evaluate the efficiency of training. It is a reference 
method for assessing the work of coaches. However, this article also has shortcomings: 1, in the research process greatly depends on expert advice and expert scoring method, which have the formation of human factors on research results, influence its objective degree. 2, the coaches are from various fields, ignoring the difference between the fields. These problems will continue to improve in the future.

\section{References}

[1] S. Zhu, "Discussion on the role of the coach: the type and quality [J]", sports teachers, (1992), p. 1.

[2] Spencer, "I'm spencer s m competence at work: models for superior performance [M]", New York john wiley\&sons inc., (1993).

[3] Mcclelland, "d c testing for competence rather than for intelligence [J]", an psy, (1973), p. 28.

[4] Y. Chen and L. Yi, "Competency research and application and development trend [J]", The management of scientific research, (2004), p. 25.

[5] R. J. Barcelona, "an analysis of the perceived competencies of recreational sport managers: toward a competency-based model for academic and professional development [C]”, university of Oregon, (2002).

[6] K. Toh, "constructing and validating competencies of sport managers instrument: a model development [C]", university of Oregon, (1998).

[7] P. M. Fokken, "construction and validation for competencies of aboard self-assessment questionnaire for park and recreation board members: a model development[C]", university of Oregon, (2004).

[8] S. M. Spencer and L. M. Spencer, "competency assessment methods [M]", London: hay/mocker research press, 1992

[9] Support staff competency dictionary [EB/OL] The department of labor. http://www.doleta.GOv/jobs.

[10] Y. Jun, "Study of elite coaches knowledge structure [J]”,Journal of Wuhan Sports Institute, (2000).

[11] F. Zhao, "Study on the status and Countermeasures of senior Basketball Coaches in China [J]", Journal of Wuhan Sports Institute, (2002).

[12] Y. Wu, "The new challenge to sports coaches [J]”, Anhui sports science and technology, (2002)

[13] J. Li and J. Chen, "Study on the overall quality of the present situation and Development Countermeasures of Guangdong Province Diving Coaches [J]”, Hubei sports science and technology, (2004).

[14] M. Wu, "A study on the competency of occupation Marketing Manager [J]", China journal net national outstanding thesis library, (2003).

[15] L. Zhong and K. Shi, "New progress of research for characteristics [J]", Nankai business review, (2003).

[16] C. Song and W. Q. Ling, "The study on the competency model of middle-level managers in Enterprises [D]", Master's thesis of Jinan University, (2003).

[17] L. Liu, "Study on the competency model of professional sports coaches [J]", sports science, (2007).

[18] L. Liu, "Research on Chinese coaches' quality based on Competency Model [J] Journal of Beijing Sport University", (2007).

[19] Y. Zhang, "Research on the performance assessment of the track and field coaches in China [J]", China sports science and technology, (2010). 
[20] L. Li, "Research on job performance structure model of professional sports coaches in China [J]", sports science, (2011).

[21] Y. Tang, "Study on Evaluation System of juvenile football coaches in Guangdong [J]", Journal of physical education, (2011).

[22] A. Charnes, W. W. Cooper and Q. L. Wei, et al. "Cone ratio data envelopment analysis and multi- objective programming [J]”, International Journal of Systems Science, vol. 20, no. 7, (1989), pp. 1099- 1118.

[23] Q. Wei, “Data envelopment analysis [M]”, Beijing: Science Press, (2004).

[24] J. Guo and D. Yang, "Overview of data envelopment analysis method [J]", Journal of Dalian University of Technology, vol. 38, no. 2, (1998), pp. 236 - 241

[25] X. Zhao, "Analysis on efficiency and effect factors of bank branches and Empirical Study on the super efficiency DEA model based on Tobit [J]”, economic science, vol. 1, (2010), pp. 85 - 96

[26] J. Wu, "Study on super efficiency DEA model based on variable returns to scale [D]", University of Science $\&$ Technology China, (2008).

[27] J. Zhang, "The DEA analysis method and model for a class of preference structure [D]", Dalian Management School of Dalian University of Technology, (1997).

[28] Z. Zhou, "A new method for evaluating the efficiency of management [J]", systems engineering, vol. 4, (1986), pp. 42-49.

[29] Q. Zhu, Z. Sheng and G. Wu, "Efficiency in DEA model [J]", Journal of Southeast University, vol. 2, (1994), pp.78 82

[30] Q. Zhu and Y. Chen, "Sensitivity research and application of data envelopment analysis [J]", Journal of system engineering, (1994).

\section{Author}

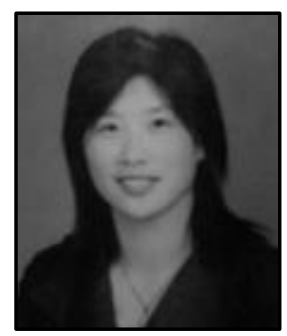

Caiyan Xu, she is a Master, majoring in Sports teaching and training. Now she is a lecturer in Jiangsu University. 
International Journal of $u-$ and e-Service, Science and Technology Vol. 7, No. 6 (2014) 